\title{
Adrenal Cyst: An Incidental Finding!
}

\author{
Amruta V Hippalgaonkar ${ }^{1}$, Chaitanya V Bokil ${ }^{2}$
}

\begin{abstract} management of adrenal cyst and briefly review the literature.

Keywords: Adrenal cyst, General anesthesia, Laparoscopy.

Research and Innovation in Anesthesia (2020): 10.5005/jp-journals-10049-0083

\section{INTRODUCTION}

Cystic adrenal lesions usually non-functioning and asymptomatic represent a rare entity and their clinical management remains controversial. ${ }^{1,2}$ The prevalence is not well characterized and is rising because of the big volume of radiologic explorations done every day. ${ }^{3}$ Adrenal incidentaloma is a term applied to an incidentally discovered adrenal mass on imaging investigation performed for reasons unrelated to adrenal pathology. ${ }^{3,4}$ This case report presents a young female patient with an incidental adrenal mass that was evaluated according to proposed recommendations and planned for laparoscopic excision.
\end{abstract}

Adrenal cysts are rare, mostly silent clinically and their management remains controversial. Herein, we report a case of adrenal cyst in a young female who was incidentally found to have a cystic lesion of either suprarenal or pancreatic origin by abdominal ultrasonography during workup for her left abdominal pain radiating to back and leg. The computed tomography (CT) revealed a large thin-walled cystic lesion, enhanced on the left suprarenal gland, with eccentric calcification, causing mass effect on the pancreas and left kidney. Laparoscopic surgical exploration was performed and the cystic adrenal mass was excised. The histopathologic diagnosis was mesothelioma. Thus, we discuss the diagnosis and

\section{Case Description}

A young 17 years old female presented with complaints of pain in the left side of the abdomen radiating to the back and left leg for 2 months, associated with nausea and fatigue. Abdominal ultrasonography revealed a cystic lesion probably of pancreatic pseudocyst or suprarenal origin. Computed tomography (CT) scan of the abdomen confirmed the presence of the left adrenal simple cyst. She had no history of any systemic illness, allergies, or taking any regular medications. On examination, the patient was conscious, comfortable, obese (body weight $75 \mathrm{~kg}$, body mass index (BMI): 35.2), vital signs were normal. The preoperative evaluation showed airway assessment of Mallampati 2, three-finger mouth opening with adequate neck movements. Systemic examination was clinically unremarkable and hence classified as American Society of Anaesthesiologists (ASA)I. Computed tomography abdomen reported large thin-walled cystic lesion, enhanced on the left suprarenal gland measuring $(6.6 \times 6.3 \times 5.8 \mathrm{~cm})$, with eccentric calcification, causing mass effect on the pancreas and left kidney. The patient had no symptoms of catecholamine excess secretion, laboratory findings including adrenal hormonal study were within the normal range. So, the mass was considered non-functioning and was scheduled for laparoscopic excision. Also, preoperative vital signs charting of the patient since admission did not reveal any persistent or episodic increase in blood pressure (BP) or blood sugar levels.

In the operating room, ASA standard monitoring was connected and two peripheral intravenous accesses were established with an $18 \mathrm{G}$ catheter in both hands. The patient was preoxygenated
${ }^{1}$ Department of Anaesthesia, Krishna Institute of Medical Sciences (KIMS), Karad, Maharashtra, India

${ }^{2}$ Department of General Surgery, Krishna Institute of Medical Sciences (KIMS), Karad, Maharashtra, India

Corresponding Author: Amruta V Hippalgaonkar, Department of Anaesthesia, Krishna Institute of Medical Sciences (KIMS), Karad, Maharashtra, India, Phone: +919769491607, e-mail: h.amruta1@ gmail.com

How to cite this article: Hippalgaonkar AV, Bokil CV. Adrenal Cyst: An Incidental Finding! Res Inno in Anesth 2020;5(1):15-17.

Source of support: Nil

Conflict of interest: None

with $100 \% \mathrm{O}_{2}$ by face mask followed by induction of anesthesia using fentanyl $100 \mu \mathrm{g}$, propofol $150 \mathrm{mg}$ (in graded doses), and vecuronium $5 \mathrm{mg}$. Mask ventilation and intubation were easy. Anesthesia was maintained with sevoflurane $2.5 \%$ in oxygen and air and intermittent vecuronium to maintain muscle paralysis. Phenylephrine, ephedrine, nitroglycerine as well as adrenaline and noradrenaline infusions were kept on standby to use in the case of emergency. Intravenous fluids are set on both peripheral lines at a total rate of $200 \mathrm{~mL} /$ hour. The patient was positioned in the right lateral position with pressure points padded. The operation commenced with the laparoscopic left lateral transabdominal approach and $50 \mu \mathrm{g}$ fentanyl given at the time of incisions. Patient BP was stable during that time within the accepted range. With the duration of 1.5 hours after incision, BP started to rise to $150 / 100$ controlled with deepening of anesthesia plane, but BP kept fluctuating with manipulation of the mass and shooting to $>170 / 120$ only once which responded to antihypertensives (labetalol bolus $5 \mathrm{mg}$ ). Thereafter, vitals were stable and the excision of the cyst was accomplished after drainage of fluid. The patient was hemodynamically stable at the end of the procedure with an estimated blood loss of $0.5 \mathrm{~L}$, total fluids are given about $3 \mathrm{~L}$, and total urine output $1.2 \mathrm{~L}$, and the duration of surgery is 3 hours. The patient was awakened, extubated uneventfully, and was transferred in a fully conscious state to the surgical intensive care unit (ICU) and discharged on the fifth postoperative day. Histopathology of the specimen confirmed mesothelioma.

() The Author(s). 2020 Open Access This article is distributed under the terms of the Creative Commons Attribution 4.0 International License (https://creativecommons. org/licenses/by-nc/4.0/), which permits unrestricted use, distribution, and non-commercial reproduction in any medium, provided you give appropriate credit to the original author(s) and the source, provide a link to the Creative Commons license, and indicate if changes were made. The Creative Commons Public Domain Dedication waiver (http://creativecommons.org/publicdomain/zero/1.0/) applies to the data made available in this article, unless otherwise stated. 


\section{Discussion}

Adrenal incidentaloma's prevalence is rising because of the big volume of radiologic explorations done for clinical conditions unrelated to adrenal disease. Most often, it consists of benignnon-functioning lesions. However, all adrenal masses should be inspected for malignancy or hypersecreting disorders. ${ }^{4,5}$

A careful history and examination are mandatory. But no comprehensive guidelines have been published by professional societies to guide the evaluation of such patients. ${ }^{5}$

Although it is possible to observe asymptomatic, nonfunctioning adrenal cysts and the role of surgery are debated, it is mandatory in the presence of symptoms, hyperfunctioning masses, suspicion of malignancy, and when the diameter is $\geq 4 \mathrm{~cm}$ or increase in cyst size. . $^{2,4,6}$

Laparoscopic management of benign adrenal cysts is safe, feasible, and effective. Unlike aspiration of cyst contents, recurrence after surgical removal is unlikely. Laparoscopic decortication and marsupialization should be the preferred treatment option for symptomatic adrenal cysts. Laparoscopic adrenalectomy is performed in cases of larger cysts. ${ }^{7,8}$ Thus, the laparoscopic technique is defined as the "gold standard" in the treatment of adrenal tumors. Compared with open techniques, the "minimally invasive" revolution will advocate attenuated surgical stress response, decreased inflammatory response, less blood loss, earlier patient recovery, a shorter hospital stay, and enhanced cosmesis. ${ }^{7,9,10}$

There is no certain published anesthetic approach; hence, it presents a challenge to anesthesiologists regarding how to best manage these patients perioperatively. To start with a thorough preoperative assessment with special attention to the history and physical examination including signs and symptoms of hormone hypersecretion becomes necessary. Similarly, the biochemical investigations carry paramount importance in deciding the plan of anesthesia and further peri-operative course.

A clinical research study demonstrates a strong association between obesity and adrenal incidentalomas. ${ }^{11}$ Also, they are characterized by the central type of fat accumulation, insulin resistance (IR), hypertension, dyslipidemia, and fatty liver disease, all being components of metabolic syndrome (MS). Thus, patients should be screened for MS during their initial workup to identify those at cardiometabolic risk and implement appropriate interventions. $^{12}$

The conduct of anesthesia is with standard induction and airway control with the tracheal tube. Full routine monitoring including core temperature measurement, end-tidal carbon dioxide $\left(\mathrm{EtCO}_{2}\right)$ should be applied. A large-bore cannula is usual. The arterial line or central venous access can be set up if a patient has comorbidities. As the procedure may be prolonged, it is sensible to use a volatile agent (desflurane or sevoflurane) for maintenance with a favorable recovery profile. It is important to ensure that pressure points are well padded. ${ }^{4}$

The position of the tracheal tube should be checked regularly. There are reports of airway obstruction during insufflation of the pneumoperitoneum, secondary to tube migration resulting from the physical movement of diaphragm and mediastinum. The physiological effects of pneumoperitoneum are numerous and can be severe. The patient may experience cardiovascular instability and hypoxemia on carbon dioxide insufflation. Endtidal carbon dioxide should be maintained at normal levels to reduce the risk of catastrophic increases in cerebral blood volume and intracranial pressure. The overall incidence of complications is low and the postoperative course is usually uneventful. Blood transfusion is hardly required as intraoperative blood loss is very low, but the patient should be carefully monitored in the immediate postoperative period for insidious hemorrhage. Multimodal analgesia should be administered. ${ }^{4}$

The patient reported, in this case, was incidentally found to have adrenal mass and was asymptomatic with normal biochemical and hormonal studies. The patient was considered obese with a BMI of 35.2 in accordance with the studies showing a strong association between obesity and adrenal incidentalomas. ${ }^{11,12}$

Although a study reported that patients with non-functioning adrenal incidentalomas exhibit higher fasting glucose as part of the MS, our patient had nearly normal blood glucose levels in the pre-op and peri-op period. This patient did not receive any antihypertensives as preoperative medications because she had no hypertension or symptoms of catecholamine secretion. Despite that intraoperative plan takes into account the high possibility of occurrence of hypertensive crisis so we had emergency drugs ready and handy.

Laparoscopic excision requires persistent tissue traction and diathermy that may cause sustained hemodynamic consequences of the same severity as during open surgery, but for a longer period of time. ${ }^{4}$ Patient-reported in this case showed hemodynamic instability with a hypertensive crisis that risks the whole outcome of the procedure and increased its risk of morbidity. However, with vigilant efforts and vigorous attempts, patient could be stabilized till the end of the procedure and successfully extubated and shifted to the surgical ICU.

\section{Conclusion}

We conclude that despite the feasibility and safety of laparoscopy in adrenal surgery and it been declared the "gold standard" method; there is a need to formulate a protocol to reach the optimum outcome in managing adrenal incidentalomas. The endocrinologist, urologist, and anesthesiologist must be incorporated in planning and taking decisions as to how to best manage such patients. However, since the researches in this field are proceeding with high evolution velocity, in the next future most of the questions that are still present should be definitively addressed. ${ }^{13}$

\section{References}

1. Tanuma Y, Kimura M, Sakai S. Adrenal cyst: a review of the Japanese literature and report of a case. Int J Urol 2001;8(9):500-503. DOI: 10.1046/j.1442-2042.2001.00359.x.

2. Koichi K, Yasukazu T, Susumu N, et al. Laparoscopic management of a complex adrenal cyst. Case Rep Urol 2015;2015:234592.

3. Huang SP, Chen CC, Li CC, et al. Adrenal cyst - a case report. Kaohsiung J Med Sci 2001;17:156-160.

4. Reema MA, Nehal G, Sherine $Q$, et al. Adrenal incidentaloma: anesthetic management, the challenge and the outcome. Anesth Essays Res 2011;5(2):217-223. DOI: 10.4103/0259-1162.94787.

5. Hevia Suárez M, Abascal Junquera JM, Boix P, et al. Management of adrenal mass: what urologists should know. Actas Urol Esp 2010;34(7):586-591. DOI: 10.1016/j.acuro.2009.11.010.

6. Lin GB, Xu M, Wang H. Diagnosis and treatment of adrenal cyst: report of 15 cases. Zhonghua Yi Xue Za Zhi 2009;89:409-411.

7. El-Hefnawy AS, El Garba M, Osman Y, et al. Surgical management of adrenal cysts: single-institution experience. BJU Int 2009;104(6):847850. DOI: 10.1111/j.1464-410X.2009.08537.x. 
8. Castillo OA, Litvak JP, Kerkebe M, et al. Laparoscopic management of symptomatic and large adrenal cysts. J Urol 2005;173(3):915-917. DOI: 10.1097/01.ju.0000152177.35204.70.

9. Guerrieri M, De Sanctis A, Crosta F, et al. Adrenal incidentaloma: surgical update. J Endocrinol Invest 2007;30(3):200-204. DOI: 10.1007/ BF03347425.

10. Hung SF, Chung SD, Chueh SC, et al. Laparoscopic management of potentially malignant or complex adrenal cysts abiding by the principles of surgical oncology. J Endourol 2009;23(1):107-113. DOI: 10.1089/end.2008.0436.
11. Kolańska K, Owecki M, Nikisch E, et al. High prevalence of obesity in patients with non-functioning adrenal incidentalomas. Neuro Endocrinol Lett 2010;31:418-422.

12. Peppa M, Boutati E, Koliaki C, et al. Insulin resistance and metabolic syndrome in patients with nonfunctioning adrenal incidentalomas: a cause-effect relationship? Metabolism 2010;59(10):1435-1441. DOI: 10.1016/j.metabol.2010.01.007.

13. Bergamini C, Prosperi P, Bruscino A, et al. Update on the laparoscopic adrenal surgery in the second decade of the century: "doubts no more? G Chir 2010;31:328-331. 